\title{
Corner rounding in EUV photoresist: tuning through molecular weight, PAG size, and development time
}

\author{
Christopher N. Anderson ${ }^{a}$, Joe Daggett ${ }^{b}$, and Patrick P. Naulleau ${ }^{a}$ \\ ${ }^{a}$ Center for X-ray Optics, Lawrence Berkeley National Laboratory \\ 1 Cyclotron Road, Berkeley, CA 94720, USA \\ ${ }^{b}$ Sumika Electronic Materials Inc, 3832 E. Watkins St. Phoenix, AZ 85034, USA
}

\begin{abstract}
In this paper, the corner rounding bias of a commercially available extreme ultraviolet photoresist is monitored as molecular weight, photoacid generator (PAG) size, and development time are varied. These experiments show that PAG size influences corner biasing while molecular weight and development time do not. Large PAGs are shown to exhibit less corner biasing, and in some cases, lower corner rounding, than small PAGs. In addition, heavier resist polymers are shown to exhibit less corner rounding than lighter ones.
\end{abstract}

Keywords: Lithography, EUV, Photoresist, Corner Rounding

\section{INTRODUCTION}

At the 2008 Extreme Ultraviolet (EUV) Symposium it was shown that corners patterned in chemically amplified (CA) photoresist via EUV $(\lambda=13.5 \mathrm{~nm})$ radiation often exhibit a rounding bias between inner and outer corners. ${ }^{1}$ In these experiments, scanning electron microscope (SEM) metrology of the photo mask showed no corner rounding bias, modeled aerial images assuming a thick mask (multilayer + absorber) revealed no corner rounding bias, and resist blur models including PROLITH, ${ }^{2}$ single blur ${ }^{3}$ and dual blur ${ }^{4}$ could not reproduce the observed bias. Today, the source of the corner rounding bias remains unknown.

In this paper, the corner rounding bias of a commercially available EUV photoresist is monitored as molecular weight, photoacid generator (PAG) size, and development time are varied.

\section{EXPERIMENTAL METHODS}

\subsection{Exposures}

All resists were exposed to EUV $(\lambda=13.5 \mathrm{~nm})$ radiation at the 0.3 numerical aperture SEMATECH Berkeley Mirofield Exposure Tool (BMET) printing facility using conventional $\sigma=0.35-0.55$ annular illumination. ${ }^{5}$ Each wafer was shot with a 9x13 focus-exposure matrix with $5 \%$ exponential dose steps and $50 \mathrm{~nm}$ focus steps.

\subsection{Features}

Dark field 700-nm elbow patterns (Figures 1a, 1b) are used for corner characterization. Inner corners are identified as those with $270^{\circ}$ of resist remaining after development (Figure 1c, left) and outer corners are identified as those with $90^{\circ}$ of resist remaining after development (Figure 1c, right).

\subsection{Metrology}

Resist metrology was carried out at LBNL on a Hitachi S-4800 SEM with a working distance of $2 \mathrm{~mm}$ and an acceleration voltage of $2.0 \mathrm{kV}$. In each corner image the SEM magnification was set to $80 \mathrm{k}$. The radius of curvature of each corner was measured using the "area fit" algorithm in an offline SEM image analysis package. ${ }^{6}$ Reported corner radius magnitude is the average radius of all seven corners in the $700 \mathrm{~nm}$ elbow pattern shown in Figure 1a. Reported corner radius uncertainty is the $3 \sigma$ standard deviation of the seven corner radii in each $700 \mathrm{~nm}$ elbow, divided by $\sqrt{7}$, which assumes independence between each corner.

For further information contact cnanderson@lbl.gov 

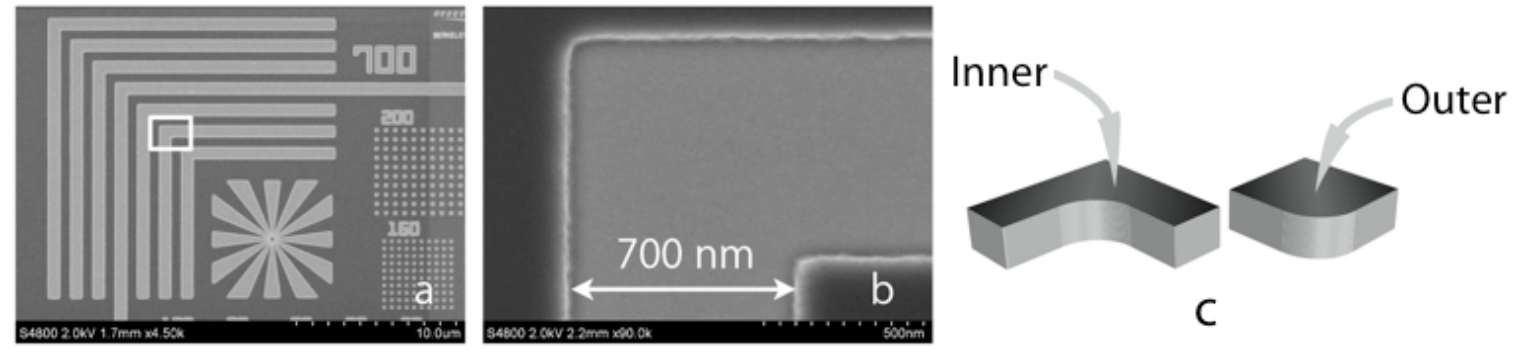

Figure 1. (a) Scanning electron microscope image of a 700-nm elbow pattern. Darker areas are the resist that remains after development; (b) zoom-in on the white box region of a; (c) visual comparison between inner and outer corners.

\section{RESISTS}

All resists were supplied by Sumika Electronic Materials, Inc. ${ }^{7}$ Two testbeds were studied. One testbed had fixed photo acid generator (PAG) size with varying polymer molecular weight. The other had fixed polymer molecular weight with varying PAG size. Resist properties for both testbeds are summarized in Table 1.

Four-inch HMDS-primed wafers were used in all experiments. Resists were spin-coated and soft baked at $110^{\circ} \mathrm{C}$ for 60 seconds to achieve a film thickness of $80 \mathrm{~nm}$. After exposure, each wafer was baked at $110^{\circ} \mathrm{C}$ for 60 seconds. Wafers were developed with a single puddle of Rohm and Haas MF26A (0.26 mol/L TMAH). Development was followed by a $60 \mathrm{sec}$ rinse with deionized water. Four development times were tested: $\ll 1 \mathrm{sec}$, $1 \mathrm{sec}, 3 \mathrm{sec}$, and $45 \mathrm{sec}$.

Table 1. Resist parameters

\begin{tabular}{lll}
\hline \hline Resist & Molecular weight & PAG size \\
\hline 1248 & Low & Mid \\
1247 & Mid & Mid \\
1246 & High & Mid \\
\hline 1250 & Low & Smalll \\
1248 & Low & Mid \\
1251 & Low & Large \\
1252 & Low & Small/Large Blend \\
\hline \hline
\end{tabular}

\section{RESULTS}

\subsection{Varying molecular weight}

Figure 2 shows the measured radii of inner and outer corners through development time: (a) is low molecular weight, (b) is mid molecular weight, and (c) is high molecular weight In summary, increasing molecular weight reduces corner rounding while maintaining approximately the same difference between inner and outer corner radii. Longer development times, in general, increase the difference between inner and outer corner radii, but only marginally.

\subsection{Varying PAG size}

Figure 3 shows the measured radii of inner and outer corners through development time for (a) small PAG, (b) mid PAG, (c) large PAG, and (d) the small/large PAG blend. In general, increasing PAG size reduces the difference between inner and outer corner radii. Also, corner rounding magnitude increases going from small PAG to medium PAG and then decreases going form medium PAG to large PAG.

unpredictably altering average corner rounding. 

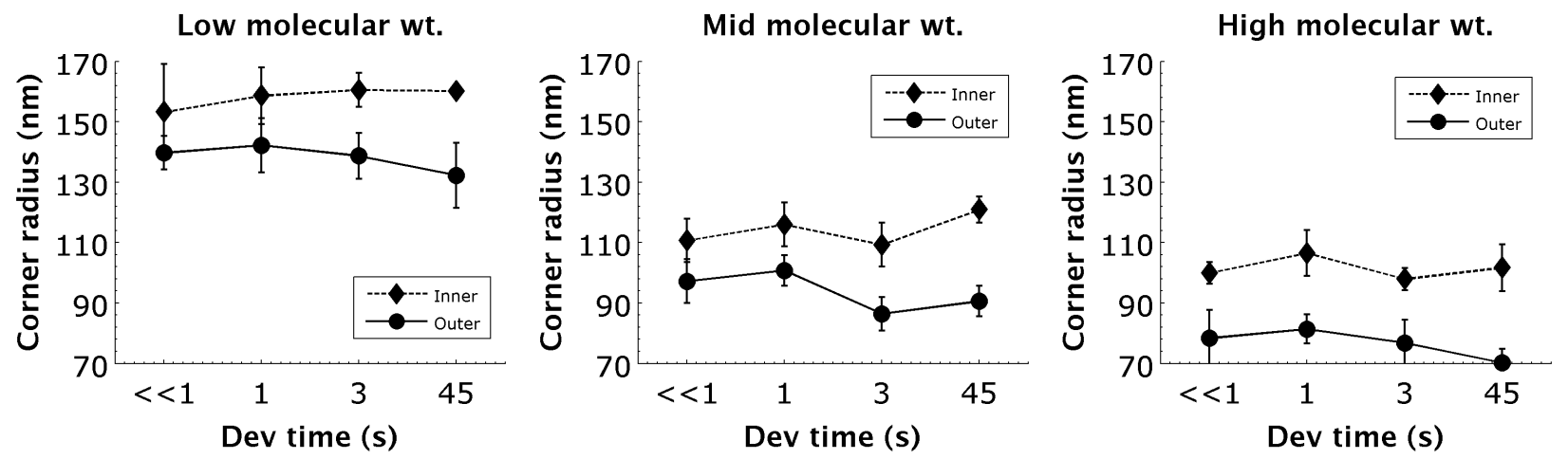

Figure 2. Corner radius vs. develop time (a) low molecular weight (b) mid molecular weight (c) high molecular weight
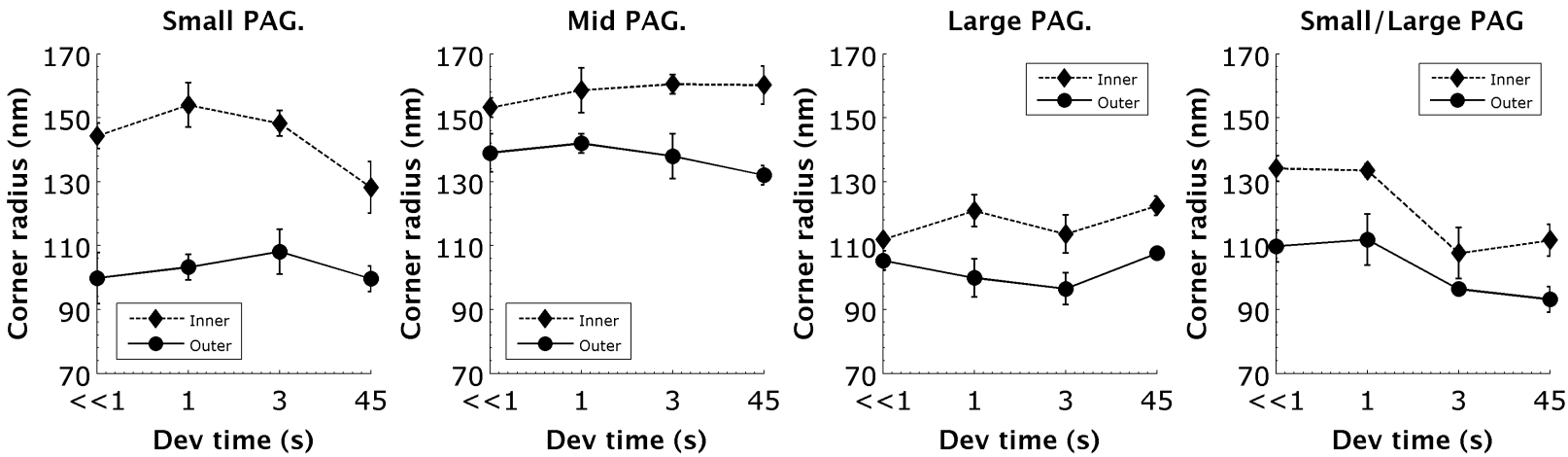

Figure 3. Corner radius vs. develop time for (a) small PAG, (b) medium PAG, (c) large PAG, and (d) the large/small PAG blend.

\section{DISCUSSION}

Figures 2 and 3 suggest different qualities about the relationship between development time and corner rounding bias. Figure 2 suggests that the bias may grow (and diverge) with development time while Figure 3, subfigures (a) and (d) show a lower bias at the longer development times. To elucidate this discrepancy, a 180 sec development time was added to the molecular weight testbed. Figure 4 is a modified version of Figure 2 that shows these additional data. The trends do not continue as expected from Figure 2. Instead, the rounding bias remains more-or-less fixed while the corner rounding magnitude drops in the low molecular weight platform and increases in the high molecular weight platform.
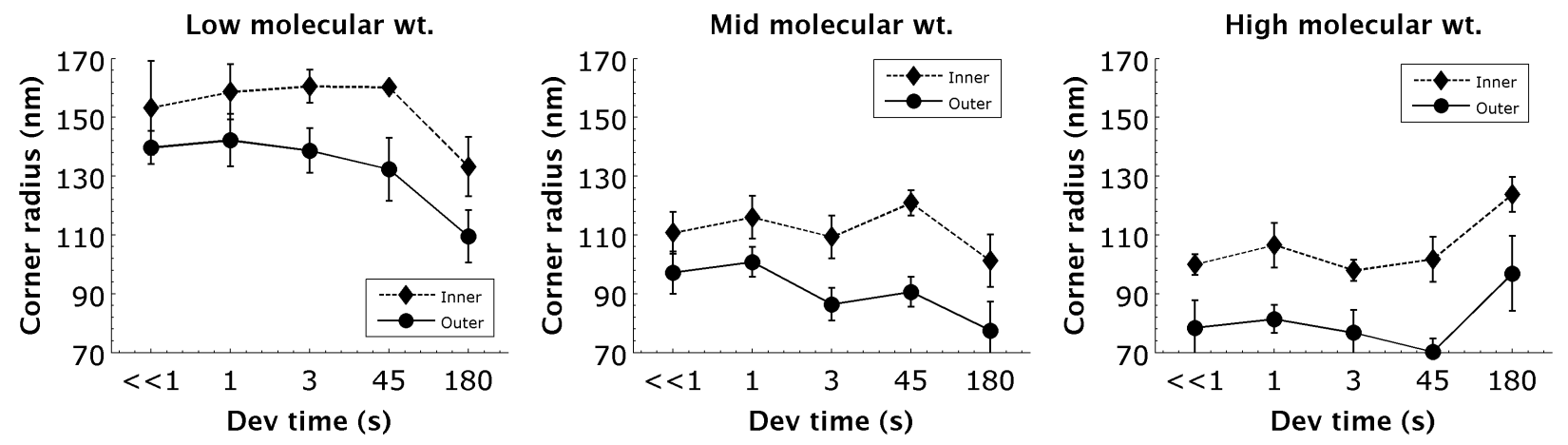

Figure 4. Corner radius vs. develop time including the added 180 second development time (a) low molecular weight (b) mid molecular weight (c) high molecular weight 
The increasing then decreasing nature of the corner rounding magnitude vs. PAG size relationship requires some attention. In 2007, Anderson, et. al. showed that average corner rounding magnitude scales with resist deprotection blur magnitude. ${ }^{8}$ Also, in 2006, Vogt et. al. showed that PAG size can affect the deprotection blur of CA photoresists. ${ }^{9}$ Within the same polymer matrix, it was found that larger PAG molecules correlate to smaller deprotection blurs. Together these results imply that larger PAGs should exhibit a lower corner rounding magnitude. In the present experiments, PAG size did influence corner rounding (which supports previous findings); however, no definite correlation between larger PAGs and lower corner rounding magnitude was found.

To test the reproducibility of these data, a small subset of the experiment (the 45 second development time, less the blended PAG formulation) was repeated on the PAG testbed using a post exposure bake (PEB) temperature of $100^{\circ} \mathrm{C}$ (reduced from $110^{\circ} \mathrm{C}$ ). Figure 5 is a modified version of Figure 3 that shows these additional data. Compared to the $110^{\circ} \mathrm{C}$ PEB data, the $100^{\circ} \mathrm{C}$ PEB data has smaller corner rounding; however, this is consistent with previous findings. ${ }^{10}$ More importantly, the trend of average corner rounding increasing from small PAG to medium PAG and then decreasing form medium PAG to large PAG remains. The mechanism driving the increasing then decreasing nature of the corner rounding magnitude vs. PAG size relationship is unknown.
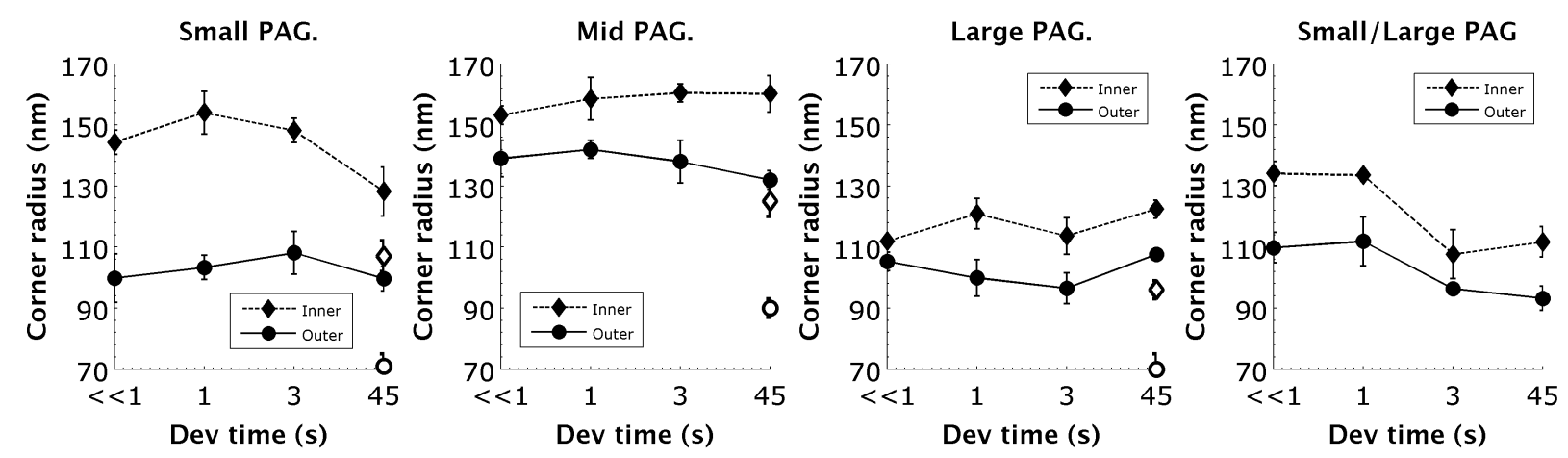

Figure 5. Corner radius vs. develop time including the added $100^{\circ} \mathrm{C}$ post exposure bake temperature data for (a) small PAG, (b) medium PAG, (c) large PAG, and (d) the large/small PAG blend.

\section{WRAP UP}

Photoacid generator (PAG) size influences corner biasing in the examined extreme ultraviolet photoresist. These data show that increasing PAG size can reduce corner biasing, and in some cases, reduce corner rounding magnitude. Development time and resist polymer molecular weight do not appear to affect corner biasing; however, increasing resist polymer molecular weight can reduce corner rounding magnitude.

\section{ACKNOWLEDGMENTS}

The authors are indebted to Paul Denham, Gideon Jones, Brian Hoef, and Ken Goldberg of the Center for X-Ray Optics (CXRO) at Lawrence Berkeley National Laboratory for expert support with the exposure tool as well as the entire CXRO engineering team for building and maintaining the exposure tool. The authors also acknowledge Nobu Ando from Sumika Electronic Materials ${ }^{7}$ for supplying resist and process support. This research was performed at Lawrence Berkeley National Laboratory using the SEMATECH-supported MET exposure facility at the Advanced Light Source. This work was supported by the Director, Office of Science, of the U.S. Department of Energy under Contract No. DE-AC02-05CH11231.

\section{REFERENCES}

1. C. Anderson and P.Naulleau. Corner rounding in photoresists for extreme ultraviolet lithography, October 2008. Oral Presentation, 2008 EUV Symposium. 
2. PROLITH is a registered trademark of KLA-Tencor, One Technology Drive, Milpitas, CA, 95035.

3. F. Houle, W. Hinsberg, M. Morrison, M. Sanchez, G. Wallraff, C. Larson, and J. Hoffnagle. Determination of coupled acid catalysis-diffusion processes in a positive-tone chemically amplified photoresist. JVST B, 18:1874-1885, 2000.

4. Y. Tanaka, Y. Kikuchi, D. Goo, H. Oizumi, and I. Nishiyama. Estimation of diffusion lengths of acid and quencher in chemically amplified resist on the basis of extreme ultraviolet exposure results. JVST B, 25(6):2114-2117, 2007.

5. P. Naulleau. Status of EUV micro-exposure capabilities at the ALS using the 0.3-NA MET optic. Proc. of SPIE, 5374:881-891, 2004.

6. SuMMIT software is distributed by EUV Technology, Martinez, CA 94553, http://www.euvl.com/summit.

7. Sumika Electronic Materials, Inc. 3832 E. Watkins St. Phoenix, AZ 85034, USA.

8. C. Anderson and P. Naulleau. Sensitivity study of two high-throughput resolution metrics for photoresists. Applied Optics, 47(1):56-63, 2008.

9. B. Vogt, S. Kang, V. Prabhu, E. Lin, S. Satija, K. Turnquest, and W. Wu. Measurements of the reactiondiffusion front of model chemically amplified photoresists with varying photoacid size. Macromolecules, 39:8311-8317, 2006.

10. C. Anderson and P. Naulleau. Do not always blame the photons: Relationships between deprotection blur, line-edge roughness, and shot noise in extreme ultraviolet photoresists. JVST B, 27(2):665-670, 2009. 